\title{
Natural Caprine Whey Oligosaccharides Separated by Membrane Technology and Profile Evaluation by Capillary Electrophoresis
}

\author{
Diana L. Oliveira $\cdot$ R. Andrew Wilbey • \\ Alistair S. Grandison • Luisa B. Roseiro
}

Received: 24 March 2013 / Accepted: 2 July 2013

(C) Springer Science+Business Media New York 2013

\begin{abstract}
The functional food market is growing rapidly and membrane processing offers several advantages over conventional methods for separation, fractionation and recovery of bioactive components. The aim of the present study was to select a process that could be implemented easily on an industrial scale for the isolation of natural lactose-derived oligosaccharides (OS) from caprine whey, enabling the development of functional foods for clinical and infant nutrition. The most efficient process was the combination of a pre-treatment to eliminate proteins and fat, using an ultrafiltration (UF) membrane of 25-kDa molecular weight cutoff (MWCO), followed by a tighter UF membrane with 1-kDa MWCO. Circa $90 \%$ of the carbohydrates recovered in the final retentate were OS. Capillary electrophoresis was used to evaluate the OS profile in this retentate. The combined membrane-processing system is thus a promising technique for obtaining natural concentrated OS from whey.
\end{abstract}

Keywords Oligosaccharides - Caprine milk whey ·

Ultrafiltration · Membrane separation · Capillary

electrophoresis profile

\section{Introduction}

The number of successful applications of membranes developed for the processing of milk and dairy products has increased markedly since the 1970s, being now the chosen

D. L. Oliveira · R. A. Wilbey • A. S. Grandison

Department of Food and Nutritional Sciences, University of

Reading, Whiteknights, Reading, RG6 6AP, UK

D. L. Oliveira $•$ L. B. Roseiro $(\square)$

Laboratório Nacional de Energia e Geologia (LNEG), Unidade de

Bioenergia, Edifício K2, Estrada do Paço do Lumiar, 22,

1649-036 Lisbon, Portugal

e-mail: luisa.roseiro@lneg.pt technique for milk fractionation and for the recovery of bioactive compounds from various feed streams (Rinaldoni et al. 2009; Akin et al. 2012).

Milk is one of the most important direct and indirect sources of oligosaccharides (OS), major components of carbohydratebased nutraceuticals. Prebiotic and anti-infective functions of milk OS, together with their pathogen receptor role, have increased the interest in these compounds (Kunz et al. 2000). In particular, caprine milk has a high level of sialylated and neutral OS, with a profile similar to that of human milk and has been reported to contain up to five and ten times more OS than bovine and ovine milks, respectively (Martinez-Ferez et al. 2006b), being a potential natural source of bioactive OS (Oliveira et al. 2012a). A growing interest in bioactive components extracted from natural resources rather than those produced synthetically arose, particularly in the case of OS, because synthetic OS are rare and expensive (Akin et al. 2012).

A few membrane-processing approaches are available for OS recovery from milk but not many refer to the recovery of natural OS from whey and even fewer from caprine origin. The earliest reports on the application of membranes for the recovery of OS can be traced back to the 1990s (Mok et al. 1995; Matsubara et al. 1996; Sarney et al. 2000). Recently, Barile et al. (2009) claimed the first study to determine the compositions of a variety of neutral and sialylated OS in bovine permeate using whey from Gorgonzola cheese production. Macedo et al. (2011) also investigated the ultrafiltration (UF) permeation performance with whey using different membranes, but in this case ovine whey was only used for the production of whey protein concentrates (WPC).

The aim of the present study was to optimise the recovery of natural OS from caprine whey, using a combination of UF methodologies in a two-stage cross-flow process. To the best of the authors' knowledge, this study is the first to use caprine whey as a natural OS source, using pilot-scale membrane technology without an enzymic pre-treatment. 